\section{RICYDE. Revista Internacional de Ciencias del Deporte doi: $10.5232 /$ ricyde \\ Rev. Int. cienc. deporte}

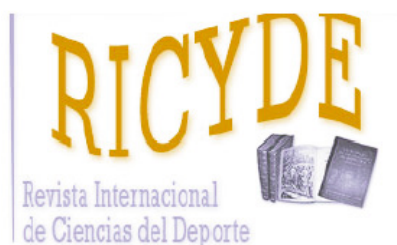

RICYDE. Revista Internacional de Ciencias del Deporte Volumen XV - Año XV

Paginas: 20-34 - ISSN: $1885-3137$

Número 55 - Enero 2019

\title{
El pensamiento del alumnado inactivo sobre sus experiencias negativas en educación física: los discursos del rendimiento, salutismo y masculinidad hegemónica \\ Inactive student thinking on their negative experiences in physical education: discourses of performance, healthism and hegemonic masculinity
}

Vicente J. Beltrán-Carrillo', José Devís-Devís²

1. Universidad Miguel Hernández de Elche. España

2. Universitat de València. España

\section{Resumen}

El propósito de este estudio es el análisis del pensamiento de los estudiantes inactivos sobre sus experiencias negativas en educación física y los discursos sociales asociados a ellas. Siete estudiantes de Bachillerato, cuatro chicos y tres chicas, clasificados como inactivos en una fase de investigación cuantitativa previa, participaron en entrevistas semiestructuradas. Cuatro temas resultaron del análisis temático de las experiencias negativas contadas por el alumnado. El primero mostraba cómo los estudiantes inactivos sufren las consecuencias de unas clases y evaluación orientadas por el profesorado hacia el rendimiento físico, especialmente los chicos obesos y las chicas con baja competencia. El segundo reflejaba la penetración del discurso de la masculinidad y salutismo en las expectativas y valoraciones del profesorado hacia su alumnado, mediatizados por posibles prejuicios corporales. El tercer tema se ocupaba de los sentimientos de incomodidad y vergüenza que vivían las chicas por exponer su cuerpo y pobre habilidad o rendimiento ante las miradas de superioridad de sus profesores y compañeros chicos. El cuarto mostraba el desprecio, la marginación y la burla experimentada por el alumnado obeso y con baja competencia y rendimiento motriz por parte de sus compañeros de clase. Este conjunto de experiencias negativas de los estudiantes inactivos aumenta la desafección hacia la actividad física y la asignatura, precisamente entre aquellos que más las necesitan. Por ello es imperioso que el profesorado reflexione en profundidad y haga frente a los discursos hegemónicos que pueden aparecer en sus clases con consecuencias negativas para su alumnado.

Key words: actividad física; salud; competencia motriz; forma física, obesidad.

\section{Abstract}

The purpose of this study is to analyze the thinking of inactive students about their negative experiences in physical education and the social discourses associated with them. Seven high school students, four boys and three girls, classified as inactive in a previous quantitative research phase, participated in semi-structured interviews. Four themes resulted from the thematic analysis of the negative experiences told by the students. The first showed how inactive students suffer the consequences of some classes and teacher-oriented assessment towards physical performance, especially obese boys and girls with low competence. The second one reflected the penetration of masculinity and healthism discourses in the teachers' expectations and valuations towards their students, mediated by possible body prejudices. The third topic dealt with the feelings of discomfort and shame experienced by the girls to expose their body and poor ability or performance under the gaze of their teachers and fellow boys. The fourth showed the contempt, the marginalization and the mockery experienced by the obese and low competence students on the part of their classmates. This set of negative experiences of inactive students, increases the disaffection towards physical activity and the subject, precisely among those who need them most. It is, therefore, imperative a depth reflection on the part of teachers in order to face the hegemonic discourses that may appear in their classes with negative consequences for their students.

Palabras clave: physical activity; health, physical competence; fitness; obesity. 
Beltrán-Carrillo, V. J., y Devís-Devís, J. (2019). El pensamiento del alumnado inactivo sobre sus experiencias negativas en educación física: los discursos del rendimiento, salutismo y masculinidad hegemónica. RICYDE. Revista internacional de ciencias del deporte. 55(15), 20-34. https://doi.org/10.5232/ricyde2019.05502

\section{Introducción}

$\mathrm{L}$ a investigación sobre el pensamiento del alumnado es un tipo o modelo de investigación que cuenta con una importante tradición en el ámbito de la enseñanza en general y la enseñanza de la educación física escolar en particular. Este modelo de investigación surgió para comprender el papel que jugaban los procesos cognitivos del alumnado en el complejo mundo de la enseñanza-aprendizaje escolar. Como señalaba Wittrock (1989), los pensamientos, creencias, afectos, opiniones y percepciones forman parte de los procesos cognitivos del alumnado que tienen repercusiones en su aprendizaje. Este modelo de investigación descansa en la idea de que la enseñanza afecta al rendimiento e implicación en las clases a través de los procesos de pensamiento del alumnado, y que el aprendizaje no se produce de forma automática a partir de la enseñanza, sino que tiene lugar a través del procesamiento activo de la información por parte de este colectivo. En general, estos planteamientos se fundamentan en el supuesto de que la acción de las personas está gobernada por su pensamiento.

Con el tiempo, la investigación ha ido incorporando factores sociales y situacionales a la cognición del alumnado (Solmon, 2006). Estas variables también median entre la enseñanza y el aprendizaje y la conducta del alumnado como, por ejemplo, la atribución, la actitud, la expectativa, la autoeficacia, la motivación y las metas. En la revisión de Lee (1997), una de las primeras sobre estudios mediacionales centrados en el alumnado de educación física, se presenta un modelo que organiza y da sentido al conjunto de investigaciones cognitivo-sociales existentes hasta ese momento. En concreto, esta autora divide los estudios en dos grupos. Uno correspondiente a las variables internas (sexo, edad, habilidad motriz y conocimientos previos) y otro grupo de variables vinculadas a la interacción social con el entorno (percepciones, actitudes, motivación, atribuciones causales y expectativas).

Desde entonces, los estudios mediacionales centrados en el pensamiento del alumnado se han dirigido a distintos objetos de estudio. Por ejemplo, unos se han ocupado de conocer las percepciones del alumnado sobre la asignatura o algunos de sus elementos curriculares (Atienza, Valencia-Peris, Martos-García, López-Pastor, y Devís-Devís, 2016; Avery y Lumpkin, 1987; Cockburn, 2000; Graham, 2008; Moreno-Murcia, Rodríguez, y Gutiérrez, 2003; Úbeda-Colomer, Monforte, y Devís-Devís, 2017), otros de profundizar en las percepciones sobre determinados aspectos curriculares ligados a la vida activa, el heterosexismo, el acoso y el miedo (Flintoff y Scraton, 2001; Monforte y Samaniego, 2017; O’Connor y Graber, 2014; Piedra, Rodríguez-Sánchez, Ries, y Ramírez-Macías, 2017) y otros de comprender las concepciones o la confusión del alumnado sobre ciertos conceptos (Hare y Graber, 2001; Hopple y Graham, 1995; Lake, 2001; Lee, Carter, y Xiang, 1995). Todos los estudios de este modelo de investigación poseen un aspecto en común que se refiere a la importancia que concede el alumnado a lo que enseña el profesorado. Es decir, considera que el punto de vista del estudiante sobre las conductas de su profesor o profesora es fundamental para entender lo que ocurre en las clases y resulta de enorme utilidad para ayudar al profesorado a mejorar su enseñanza.

En este trabajo, en particular, nos ocupamos del pensamiento del alumnado sobre sus experiencias, situándonos en la tradición investigadora iniciada por Carlson (1995), Portman (1995) y Walling y Martinek (1995) que se ocupaban de las experiencias negativas en la educación física escolar. Desde entonces, otros trabajos que profundizan en las experiencias negativas de alumnos/as en la asignatura han visto la luz (Brooks y Magnusson, 2006; Ennis, 1996; Spencer-Cavaliere y Rintoul, 2012; Van Daalen, 2005). No obstante, este estudio va más allá de los estudios mediacionales de influencia cognitivo-social que centran su atención en las interacciones que ocurren en las clases de educación física. Como señalara Sicilia (2003), una 
Beltrán-Carrillo, V. J., y Devís-Devís, J. (2019). El pensamiento del alumnado inactivo sobre sus experiencias negativas en educación física: los discursos del rendimiento, salutismo y masculinidad hegemónica. RICYDE. Revista internacional de ciencias del deporte. 55(15), 20-34. https://doi.org/10.5232/ricyde2019.05502

perspectiva mediacional muy cerrada puede dificultar una comprensión compleja de los procesos de enseñanza-aprendizaje que sea más ecológica y que considere las interpretaciones del alumnado dentro de una red amplia de significados que comparte todo el grupo social estudiado. Por ello, nuestro enfoque conecta las percepciones surgidas del entorno interactivo de las clases con aspectos socioculturales más amplios. Así lo han abordado trabajos más recientes que ponen el foco de atención en grupos marginados y vulnerados en educación física y deporte (Beltrán-Carrillo, Devís-Devís, Peiró-Velert, y Brown, 2012a; Devís-Devís, PereiraGarcía, López-Cañada, Fuentes-Miguel, y Pérez-Samaniego, 2018; Fisette, 2013; O’Sullivan y MacPhail, 2010; Rich, De Pian, y Francombe-Webb, 2015). Para conseguir esta conexión, este artículo se apoya especialmente en el concepto de discurso social que también invade la escuela y la educación física escolar. Desde el punto de vista sociológico, el término discurso hace referencia a un conjunto de ideas relativamente consistente, construido socialmente, que la gente utiliza para desenvolverse en la vida social y dotar de sentido a sus experiencias (Denison y Scott-Thomas, 2011). Podría decirse que los discursos sociales establecen principios, parámetros, o símbolos y significados compartidos que influyen en el modo en que las personas entienden e interpretan la realidad social, en lo que las personas piensan de sí mismas (identidad) y en el modo en que se comportan en sus vidas (Beltrán-Carrillo, Devís-Devís, y Peiró-Velert, 2018).

Los discursos sociales van más allá de la comunicación de conocimiento e implican prácticas sociales y relaciones de poder entre las personas (Garrett, 2004a). Los discursos cambian a lo largo del tiempo y compiten con otros en diferentes contextos sociales. Además, pueden ser reproducidos, asumidos, o rechazados en mayor o menor medida, ya que cada individuo representa un agente activo que interacciona con los discursos sociales y se ve influido por éstos de diferente forma (Johnson, Gray, y Horrell, 2013).

Por lo tanto, el objetivo de este estudio consiste en analizar el pensamiento de un grupo de estudiantes inactivos sobre sus experiencias negativas en educación física y los discursos sociales con los que se encuentran relacionadas. De esta manera podremos entender por qué surgen estas experiencias y cuál es su impacto en la subjetividad del alumnado, y lo más importante, podremos mejorar nuestra actividad docente como profesionales de la educación física escolar. Los referentes teóricos que se describen en el apartado siguiente nos permiten acercamos al pensamiento del alumnado desde una perspectiva sociocultural. Es decir, considerando que sus pensamientos reflejan y, a la vez, son influidos por los discursos sociales que les afectan en su día a día en las clases de educación física y tienen consecuencias para su vida presente y futura.

\section{Los discursos sociales del rendimiento, el salutismo y la masculinidad hegemónica en educación física.}

En el seno de las sociedades neoliberales, sustentadas en valores propios de la economía de mercado como el individualismo, la competitividad, la productividad, la eficiencia, el consumismo o el materialismo (Marsi, 2007; Olssen y Peters, 2005), existen diversos discursos sociales interrelacionados que pueden tener una importante repercusión en la educación física escolar.

En primer lugar, destacaremos lo que podemos denominar discurso del rendimiento. En diferentes contextos sociales como, por ejemplo, determinados ámbitos del mundo empresarial, académico o deportivo, el rendimiento es el principal propósito y valor. Un rendimiento entendido como productividad o eficiencia para conseguir resultados concretos y objetivos (por ejemplo, lograr más ventas de un producto, publicar más artículos científicos o mejorar una marca deportiva). En estos contextos, la valía o calidad de una persona depende del rendimiento 
Beltrán-Carrillo, V. J., y Devís-Devís, J. (2019). El pensamiento del alumnado inactivo sobre sus experiencias negativas en educación física: los discursos del rendimiento, salutismo y masculinidad hegemónica. RICYDE. Revista internacional de ciencias del deporte. 55(15), 20-34. https://doi.org/10.5232/ricyde2019.05502

que puede aportar, a la vez que existe un sistema de recompensas y sanciones que genera competitividad y presión para cumplir con los estándares (Ball, 2003). De forma semejante, la educación física se convierte en ocasiones en un contexto de rendimiento en el que la competencia motriz, el rendimiento físico, la competitividad, o la victoria acaban siendo más importantes que el aprendizaje, la salud, la diversión o la inclusión social (Beltrán-Carrillo et al., 2012a). En educación física, el rendimiento, habilidad, forma física y cuerpo del alumnado está constantemente expuesto, siendo evaluado por el profesorado y sus iguales. A su vez, algunos alumnos/as mostrarán una elevada competencia, rendirán bien en las tareas de clase y conseguirán las respectivas recompensas (éxito, reconocimiento, diversión, respeto, buena calificación, etc.), mientras que otros mostrarán una baja competencia y recibirán las sanciones propias de un bajo rendimiento (fracaso, incomodidad, vergüenza, rechazo, acoso, infravaloración, peor calificación, etc.). Esta situación parece contradictoria, ya que supuestamente todo el alumnado, no sólo los que presentan más competencia motriz y forma física, debería tener la oportunidad de disfrutar de los beneficios que la educación física debe aportar (aprendizaje motor, educación en valores sociales positivos, promoción de un estilo de vida activo y saludable, etc.).

El segundo discurso social que puede influir en la educación física escolar sería el salutismo, término acuñado por Crawford (1980). El salutismo es un discurso que relaciona diversas creencias y supuestos socialmente compartidos sobre el cuerpo, la actividad física y la salud (Devís-Devís, 2014; Kirk y Colquhoun, 1989). Más recientemente también se ha relacionado con la percepción de riesgos para la salud derivados de la inactividad física y la obesidad (Johnson, Gray y Horrell, 2013; Rich et al., 2015). El salutismo está estrechamente ligado al individualismo, ya que concibe la salud como una cuestión de responsabilidad y elección personal (Rich et al., 2015), obviando el efecto de factores genéticos, sociales y ambientales en la salud de las personas. Por lo tanto, este discurso conlleva una dimensión moral. Aquellas personas que no exhiben una buena salud, como las que muestran sobrepeso u obesidad, pueden ser consideradas personas irresponsables, perezosas y con falta de disciplina, porque podrían haber evitado su condición controlando su dieta y realizando más ejercicio físico (BeltránCarrillo et al., 2018; Rich et al., 2015). El salutismo también sienta sus raíces en el materialismo característico de una sociedad de mercado. Muestra de ello es que este discurso concibe el cuerpo en forma, delgado y musculado, como un indicador de forma física y salud, mientras que la grasa se estigmatiza y la gordura se concibe como un problema para la salud, el bienestar, la productividad o la competitividad (Mansfield y Rich, 2013). Se trata de una visión superficial que se queda en la forma corporal, como dimensión más material, objetiva y tangible del ser humano. De este modo, con el salutismo emergen asociaciones acríticas y simplistas entre la salud y la forma corporal (Beltrán-Carrillo et al., 2018). Por ejemplo, creer que toda persona con un cuerpo delgado/musculado está sana y toda persona con sobrepeso tiene problemas de salud, cuando concepciones estereotipadas como ésta han sido demostradas científicamente como incorrectas (Campos, Saguy, Emsberger, Oliver, y Gaesser, 2006). Además, el salutismo puede promover la insatisfacción corporal, la vergüenza, la marginación y los trastornos del comportamiento alimentario y de ejercicio de aquellos que no simbolizan el cuerpo sano y en forma (Johnson et al., 2013; Mansfield y Rich, 2013).

No obstante, la construcción social de lo que se concibe como cuerpo ideal no solo se ve influida por el salutismo. Las ideas relativas al género también poseen una enorme influencia en los discursos sobre el cuerpo ideal, promoviendo el rechazo social a la grasa, la delgadez en las mujeres y la musculatura en los hombres (Garrett, 2004b; Johnson et al., 2013; Scraton y Flintoff, 2002). Estas visiones de género no sólo están relacionadas con la forma física y la salud, sino también con la juventud, la sensualidad y la belleza, tan importantes en una sociedad 
Beltrán-Carrillo, V. J., y Devís-Devís, J. (2019). El pensamiento del alumnado inactivo sobre sus experiencias negativas en educación física: los discursos del rendimiento, salutismo y masculinidad hegemónica. RICYDE. Revista internacional de ciencias del deporte. 55(15), 20-34. https://doi.org/10.5232/ricyde2019.05502

de consumo en la que el cuerpo ideal se utiliza para publicitar productos y servicios (Garrett, 2004b; Shilling, 2012). Una sociedad de consumo en la que, a su vez, el cuerpo es un receptor de consumo, cuando mucha gente, en busca del cuerpo ideal, recurre a productos y servicios para mejorar su cuerpo (cirugía estética, cremas antiarrugas, pastillas que supuestamente favorecen la pérdida de peso, servicios de nutrición, gimnasios, etc.).

En este sentido, poseer un cuerpo adecuado o inadecuado, según estos discursos hegemónicos, se ha convertido en algo muy importante en la sociedad contemporánea. Esto se debe a que, tal como Shilling (2012) indica, el cuerpo posee valor simbólico y es una forma de capital físico que puede dar acceso a beneficios simbólicos y materiales (poder, estatus social, alta autoestima, mejores posibilidades laborales, etc.). Según la apariencia física y el valor simbólico del cuerpo, algunos cuerpos y algunas personas tienden a ser más valoradas que otras (Adelman y Ruggi, 2008). Por lo tanto, en educación física el alumnado con un cuerpo ideal puede ser más valorado, mientras que aquellos que se alejan del cuerpo ideal pueden sufrir la infravaloración del profesorado y de sus compañeros y compañeras.

Por último, las ideas y prácticas sociales relacionadas con la masculinidad hegemónica representan otro discurso que puede influir en la educación física escolar. Connell (2005) define la masculinidad hegemónica como la forma de masculinidad que es culturalmente exaltada en la sociedad y ocupa la posición hegemónica en las relaciones de género. Los hombres que representan la masculinidad hegemónica no solo tienden a dominar a las mujeres, sino también a cualquier hombre que no sea un verdadero hombre según sus concepciones. La masculinidad hegemónica se relaciona con el cuerpo atlético y musculado (mesomorfo), la heterosexualidad, la fortaleza física, el poder, la competencia, el éxito, la competitividad, la neutralidad emocional, la racionalidad y el individualismo (Bramham, 2003; Connell, 2005; Kenway y Fitzclarence, 1997). Por lo tanto, la masculinidad hegemónica se distancia de la debilidad física, la emoción, la dependencia, la cooperación, la empatía o la compasión. En ocasiones, la educación física puede convertirse en un contexto de rendimiento, en el que se valore especialmente la fuerza, la velocidad y la competitividad, quedando bajo una clara dominación masculina (Beltrán-Carrillo et al., 2012a). En estos casos, las chicas y los chicos que no representen los valores de la masculinidad hegemónica pueden acabar siendo infravalorados, rechazados o marginados en educación física.

\section{Método}

\section{Participantes}

Los participantes de este estudio cualitativo colaboraron en dos fases de una investigación sobre los patrones de actividad física en la adolescencia y los factores socio-culturales que influyen en la actividad/inactividad física. El diseño, procedimiento y consideraciones éticas de este proyecto de investigación fueron aprobados por la Generalitat Valenciana, institución que a su vez financió el estudio.

La primera fase de esta investigación consistió en una encuesta centrada en la medición de los niveles de actividad física y gasto energético de 395 adolescentes (Beltrán-Carrillo, DevísDevís, y Peiró-Velert, 2012b; Peiró-Velert, Valenciano-Valcárcel, Beltrán-Carrillo, y DevísDevís, 2014). Los participantes tenían 17-18 años y cursaban bachillerato en 12 centros educativos diferentes de la Comunidad Valenciana. Según sus niveles de actividad física y consiguiente gasto energético, fueron clasificados en activos, moderadamente activos, inactivos y muy inactivos. En la segunda fase de la investigación, los adolescentes clasificados en las dos primeras categorías fueron definidos como activos y los clasificados en las dos últimas como inactivos. Esta segunda fase consistió en un estudio cualitativo centrado en recopilar 
Beltrán-Carrillo, V. J., y Devís-Devís, J. (2019). El pensamiento del alumnado inactivo sobre sus experiencias negativas en educación física: los discursos del rendimiento, salutismo y masculinidad hegemónica. RICYDE. Revista internacional de ciencias del deporte. 55(15), 20-34. https://doi.org/10.5232/ricyde2019.05502

información sobre los factores personales y socio-culturales relacionados con la participación en actividad física de los adolescentes, que derivó en varias publicaciones (Beltrán-Carrillo et al., 2012a; Beltrán-Carrillo et al., 2018; Devís-Devís, Beltrán-Carrillo y Peiró-Velert, 2015). La información recopilada sobre las experiencias negativas de los adolescentes en la educación física escolar sirvió de base para el presente artículo. Un total de 20 adolescentes de nueve centros educativos diferentes fueron entrevistados (siete chicos y seis chicas eran activos, mientras que cuatro chicos y tres chicas eran inactivos).

\section{Procedimiento y recolección de datos}

Los datos cualitativos de este estudio se obtuvieron mediante entrevistas semiestructuradas, cuya duración estuvo comprendida entre los 45 minutos y una hora. El entrevistador estableció contacto telefónico con aquellos adolescentes que habían expresado, en la primera fase de la investigación, su disponibilidad para ser entrevistados en una segunda fase. Todos los adolescentes que decidieron participar contaron con la autorización de sus padres/madres, a los que se les ofreció la oportunidad de estar presentes en la entrevista si así lo preferían. Las entrevistas se realizaron donde sugerían los participantes y sus familiares. Normalmente tuvieron lugar en los hogares de los adolescentes, aunque en alguna ocasión se realizaron en una cafetería o en un banco de un parque público.

El grupo final de informantes contó con más adolescentes activos que inactivos y con más chicos que chicas. Esto se debió a que los adolescentes inactivos mostraron menos interés por un estudio relacionado con la participación en actividad física y deporte. Respecto a las chicas, algunas afirmaron sentir cierta vergüenza ante el hecho de ser entrevistadas por un hombre que no conocían, a la vez que algunas madres expresaron cierta desconfianza.

Con el objetivo de ganarse la confianza de los adolescentes y sus familias, el entrevistador mostró su Documento Nacional de Identidad y un certificado que demostraba su vinculación oficial con el proyecto de investigación. Los participantes también fueron informados de su derecho a no contestar preguntas que pudieran incomodarles y a abandonar la entrevista en cualquier momento. El consentimiento para realizar la entrevista fue registrado al inicio de la grabación. Además, el entrevistador garantizó la confidencialidad de los datos y el anonimato de los informantes se preservó utilizando seudónimos.

\section{Análisis de datos}

Las entrevistas fueron transcritas y posteriormente analizadas con la ayuda del software Nvivo (Bazeley y Jackson, 2013), que se utilizó para almacenar, organizar y clasificar la información de manera eficiente. La estrategia de análisis utilizada fue el análisis temático (Braun y Clarke, 2006). En primer lugar, se realizó una lectura profunda de las transcripciones para adquirir una idea global de la información que contenían. Posteriormente, se codificaron los fragmentos de texto que representaban experiencias $\mathrm{u}$ opiniones negativas de los adolescentes relacionadas con la educación física. Se comprobó que estas experiencias y opiniones negativas estaban asociadas a la falta de competencia motriz o de forma física, al género y a la posesión de determinadas formas corporales. Entonces se recurrió a la literatura científica en busca de referentes teóricos que sirvieran para analizar, interpretar y dar sentido a los datos (ver apartado de marco teórico). Con el marco teórico de referencia se identificaron los temas que figuran en el siguiente apartado y se procedió a la redacción de su contenido. En este sentido, la fase de redacción debe ser reconocida como una parte fundamental del análisis temático, ya que durante la misma se refina el análisis, así como las ideas que contiene, y se busca una narración que ofrezca una clara interpretación de los datos (Braun y Clarke, 2006; Sparkes y Smith, 2014). 
Beltrán-Carrillo, V. J., y Devís-Devís, J. (2019). El pensamiento del alumnado inactivo sobre sus experiencias negativas en educación física: los discursos del rendimiento, salutismo y masculinidad hegemónica. RICYDE. Revista internacional de ciencias del deporte. 55(15), 20-34. https://doi.org/10.5232/ricyde2019.05502

El proceso de análisis de datos seguido en este artículo fue liderado por el primer autor, mientras que el segundo autor ejerció el rol de amigo crítico (Smith y McGannon, 2017), revisando el proceso de análisis e intercambiando ideas y reflexiones con su compañero. La labor del amigo crítico sirvió parar mejorar la calidad de las interpretaciones, así como el rigor y la credibilidad del análisis de datos.

\section{Resultados y discusión}

Es de destacar que todas las opiniones negativas relacionadas con la educación física identificadas en los datos fueron narradas exclusivamente por adolescentes inactivos. En los siguientes apartados se describen, interpretan y discuten los temas que derivan del análisis de los datos.

\section{Sufrir la valoración del rendimiento físico en educación física}

Algunos alumnos consideraban que era injusto que los profesores (hombres) de educación física evaluaran, desde una perspectiva pseudoigualitaria o igualitarista, a todo el alumnado con los mismos estándares en pruebas de condición física o habilidad motriz:

Andrés: El profesor de educación física que he tenido, a la hora de pedir, nos pedía a todos por igual... Yo me acuerdo que iba puteado, hablando claro, porque iba siempre a remolque de lo que hacíamos todos. Por ejemplo,... yo a lo mejor pesaba 90 ó 95 kilos, y había un tío que pesaba 78, y me pedía que corriera tanto como el de 78, y yo decía: "ipero dónde vas, pero si no puedo yo correr tanto para qué me pides!”... Al menos otro modo de evaluar no... porque decía que todos por igual, y era todos por igual, 'Si guana' y va que chuta. También depende de profesores, pero el que lo aplica así podría cambiarlo (chico inactivo).

Sara: A lo mejor tú no quieres hacer algo y... el otro [profesor de educación física] está ahí: “¡Pues si no lo haces vas a suspender!”, en cuanto ya te presionan... [...] Yo no sé dar la voltereta.... el profesor como sabe hacerlo pues se piensa que para todo el mundo es igual de fácil...y yo aunque lo intente... (chica inactiva).

En ocasiones, el profesorado de educación física, influido por el extendido discurso social del rendimiento, puede entender que la manera más justa y adecuada de evaluar y calificar es valorando el rendimiento físico, concreto y objetivo, en pruebas de condición física y habilidad motriz. Esta manera de evaluar parece estar influida por el mundo del deporte, donde el rendimiento es un valor supremo, y es totalmente frecuente medir, cronometrar, tabular resultados y ofrecer rankings (López-Pastor et al., 2006; López-Pastor, Kirk, Lorente-Catalán, MacPhail y Macdonald, 2013). Según los autores citados anteriormente, la predilección por este sistema de evaluación puede deberse, entre otros motivos, a que representa una solución fácil, cómoda, neutral y objetiva al problema de la evaluación/calificación, dando menos margen a discusiones y reclamaciones.

Sin embargo, rendir más en pruebas concretas de condición física y habilidad motriz no tiene por qué significar que los alumnos han aprendido más o que han alcanzado las diversas competencias que pueden adquirirse en educación física (López-Pastor et al., 2006). Respecto a las competencias relacionadas con la salud y la adquisición de un estilo de vida activo y saludable (Real Decreto 1105/2014), que un alumno rinda muy bien en un test de velocidad o resistencia no implica necesariamente que sepa evaluar sus cualidades físicas, o diseñar y poner en práctica un programa personal de actividad física saludable. Además, este sistema de evaluación suele impactar negativamente en los alumnos menos habilidosos y con menos forma física, que sienten la presión para cumplir con estándares a los que difícilmente pueden llegar 
Beltrán-Carrillo, V. J., y Devís-Devís, J. (2019). El pensamiento del alumnado inactivo sobre sus experiencias negativas en educación física: los discursos del rendimiento, salutismo y masculinidad hegemónica. RICYDE. Revista internacional de ciencias del deporte. 55(15), 20-34. https://doi.org/10.5232/ricyde2019.05502

y reciben las sanciones por no tener éxito en un contexto de rendimiento (infravaloración, percepción de fracaso, baja calificación, burlas, etc.).

\section{Masculinidad, salutismo y alumnos valorados/infravalorados}

Según los comentarios de algunas adolescentes, ciertos profesores (hombres) mostraban favoritismo hacia los chicos y hacia aquellos alumnos con un cuerpo atlético:

Sara: ...los profesores de gimnasia suelen tener mucho favoritismo, por ejemplo, en nuestra clase suelen poner mucha más nota si eres chico, que si eres chica. Y luego siempre se fija en el cuerpo que tienes, si eres así como más atlético o lo que sea te pone más nota que si... Yo pienso que no tiene nada que ver... [lo que importa es el] esfuerzo... no puedes dar más (chica inactiva).

En línea con el comentario de Sara, estudios previos también han identificado este favoritismo hacia los alumnos chicos por parte de profesores de educación física (hombres), así como el bajo interés y bajas expectativas que muestran respecto a las habilidades y competencias que las chicas pueden mostrar en la asignatura (Fisette, 2013; Flintoff y Scraton, 2001; Van Daalen, 2005). Estas actitudes y comportamientos de los profesores varones parecen vinculados al discurso de la masculinidad hegemónica. No obstante, el profesorado de educación física también puede verse al mismo tiempo influido por el salutismo. Es cierto que la promoción de la salud a través de un estilo de vida activo es un objetivo fundamental de la educación física (Real Decreto 1105/2014). Incluso gran parte del profesorado llega a considerarlo el principal propósito de la educación física, siendo conscientes de los riegos del sedentarismo y la obesidad en la sociedad actual (Albarracín-Pérez, Moreno-Murcia, y Beltrán-Carrillo, 2014). Sin embargo, el profesorado influido por el discurso del salutismo y su moral individualista podría llegar a tener ciertos prejuicios hacia el alumnado cuyo cuerpo no simboliza la forma física y la salud, como los alumnos/as con sobrepeso u obesidad. Este profesorado podría entender que el alumnado que posee esos cuerpos inadecuados son alumnos/as irresponsables por no cuidar su salud, no controlar su dieta, mantener un estilo de vida sedentario, no tener interés por el deporte y no esforzarse en las actividades físico-deportivas. De hecho, estudios previos han mostrado que el profesorado de educación física muestra actitudes más negativas y expectativas de habilidad más bajas hacia el alumnado con sobrepeso en comparación con el alumnado que no posee sobrepeso (Peterson, Puhl, y Luedicke, 2012).

\section{Incomodidad y vergüenza ante la exposición pública del cuerpo y su motricidad}

Como se ha comentado, en ocasiones la educación física se convierte en un contexto de rendimiento físico, dominado principalmente por los hombres (profesores de educación física y alumnos chicos), en el que unas formas corporales son más valoradas que otras. En estos casos, es entendible que algunas alumnas confesaran sentir incomodidad y vergüenza cuando tenían que realizar alguna tarea motriz en la que no se sentían especialmente competentes:

Marta: A mí, como soy un poco cortada, lo que menos me gustaba era lo típico de estar haciendo abdominales, uno por uno, y que los demás te estuvieran viendo...¿¿sabes?...todos al montón... a mí me daba vergüenza...eso, delante los chicos que iban muy bien en educación física o chicas que iban muy bien, porque...yo que sé, a mí no me da vergüenza hacer nada delante de mi grupo, pero otras personas que son... super...que se creen superiores a mí... (chica inactiva).

Esta problemática ha sido tradicionalmente identificada en la literatura. Diferentes estudios han destacado que muchas chicas se sienten inseguras, incómodas y avergonzadas cuando se sienten observadas en educación física, particularmente bajo la mirada de los chicos (Garret, 2004b; Fisette, 2013; Flintoff y Scraton, 2001; Van Daalen, 2005). Este recelo ante la exposición 
Beltrán-Carrillo, V. J., y Devís-Devís, J. (2019). El pensamiento del alumnado inactivo sobre sus experiencias negativas en educación física: los discursos del rendimiento, salutismo y masculinidad hegemónica. RICYDE. Revista internacional de ciencias del deporte. 55(15), 20-34. https://doi.org/10.5232/ricyde2019.05502

pública del cuerpo y su motricidad frente a los compañeros puede estar relacionado con experiencias previas de marginación en educación física, como las que se describen en el siguiente apartado.

\section{Bajo rendimiento fisico, obesidad y alumnos marginados}

Algunos alumnos/as inactivos con obesidad, baja forma física o baja competencia motriz, relataron haber sufrido desprecio y marginación por parte de sus compañeros en las clases de educación física. Curiosamente, se referían a los chicos en forma y habilidosos como principales responsables de esas actitudes y comportamientos de rechazo y burla:

Andrés: [Alumno con obesidad en la etapa de educación primaria] Me acuerdo, por ejemplo, en primaria... era simplemente salir, pegar dos o tres vueltas a los campos de fútbol y luego, a jugar a lo que sea. Siempre ibas tú el último, que no podías con tu alma, y te pasaban... “¡Eh, venga va! ¡A ver si movemos el culo!”. Pues eso te hace sentirte mal [...] siempre había alguno que te hacia la puñeta, como es lógico...a lo mejor tú estabas corriendo y él te decía: ¡ah!, ¡Qué culazo!... y a mí no me hacía gracia... y aquel siempre presumiendo de lo buen competidor que era... iguahh! Soy el mejor... eso me repatea (chico inactivo).

Laura: No me gustaban las clases de educación física... de pequeña menos aún... seguramente... porque de pequeños, los niños son más crueles, y que yo tuviera poca facilidad para todo esto... y entonces pensaba: '¡ahora me toca la clase que peor se me da!'... no iba muy a gusto a clase... como son los niños que se meten los unos con los otros... 'yo soy el mejor en esto [se refiere a chicos] y...voy a meterme con los demás'...yo no era una de las personas con las que más se metían, pero... con poco que me digan ya... [sonríe], ya hacía daño, entonces...[tono serio en la voz], no sé no... es que no me gustaba en sí la asignatura. Quizá por eso me guste ahora menos... (chica inactiva).

Estudios previos con niños y adolescentes han demostrado resultados similares. Por ejemplo, el estudio de Li y Rukavina (2012) indicó que el alumnado con sobrespeso suele sufrir provocaciones y burlas en educación física. En la investigación de O’Connor y Graber (2014), se constató que aquellos alumnos que eran percibidos como diferentes por parte de sus compañeros, ya fuera por su apariencia física, tamaño corporal, o escasa habilidad física, eran los que recibían episodios de acoso y marginación en educación física. Incluso el estudio de Jachyra (2016) nos advierte que una educación física basada en los valores de la masculinidad hegemónica, la competitividad y el favoritismo hacia los chicos habilidosos, con cuerpos musculados y en forma, es el semillero de graves situaciones de rechazo, burla y acoso escolar hacia aquellos niños y adolescentes que no representan los valores hegemónicos de estos contextos. Lo más preocupante es que estos estudios señalan al profesorado como parte del problema. Unas veces por no percatarse de este tipo de situaciones, por omisión, o por no saber cómo intervenir. Otras veces porque son los primeros representantes y promotores de los discursos hegemónicos y excluyentes que a veces imperan en educación física.

Por último, cabe destacar que el caso de Andrés ilustra cómo la combinación de los discursos que rondan en torno al rendimiento, la masculinidad, el salutismo y el cuerpo ideal pueden ejercer un efecto especialmente dañino en algunos alumnos/as. Parece que cuando un alumno/a con un cuerpo obeso muestra además baja forma física o competencia motriz, una particular combinación negativa tiene lugar, atrayendo el rechazo y la marginación de los demás. Respecto al caso de Laura, ilustra la relación que existe entre las experiencias negativas y la desafección con la asignatura de educación física, que podría acabar derivando en la predisposición hacia un estilo de vida sedentario. De hecho, probablemente no fue casualidad 
Beltrán-Carrillo, V. J., y Devís-Devís, J. (2019). El pensamiento del alumnado inactivo sobre sus experiencias negativas en educación física: los discursos del rendimiento, salutismo y masculinidad hegemónica. RICYDE. Revista internacional de ciencias del deporte. 55(15), 20-34. https://doi.org/10.5232/ricyde2019.05502

que todas las experiencias y opiniones negativas reflejadas en este estudio fueron informadas por adolescentes inactivos.

\section{Conclusiones e implicaciones}

Este estudio cualitativo ha analizado el pensamiento de un grupo de adolescentes inactivos sobre sus experiencias negativas en educación física, influidas por los discursos del rendimiento, el salutismo y la masculinidad hegemónica. Los datos sugieren que, en algunos centros educativos, el alumnado inactivo con baja forma física, baja competencia motriz, sobrepeso u obesidad, es presionado, infravalorado o marginado en educación física. Esta situación probablemente suceda porque este alumnado representa lo inapropiado en educación física, según ciertos discursos sociales que subyacen en el origen y la pervivencia de contextos sociales excluyentes e injustos. Parece lógico pensar que este tipo de vivencias acaben influyendo negativamente en la identidad de estos alumnos/as (falta de autoestima, complejos corporales, baja percepción de competencia), en su rechazo a la educación física y en la consolidación de un estilo de vida sedentario. En este sentido, resulta contradictorio que aquellos alumnos/as que más necesitan de los supuestos beneficios de la educación física (aprendizaje motor, educación en valores sociales positivos, promoción de la salud, diversión, etc.) salgan perjudicados de estos contextos.

Es imprescindible que el profesorado de educación física reflexione en profundidad y haga frente a los discursos hegemónicos que pueden acabar manifestándose en sus clases $\mathrm{y}$ provocando consecuencias negativas. Sería deseable que la promoción de un entorno inclusivo en las clases fuera un objetivo inicial y prioritario para el profesorado, de modo que se respetara a todo el alumnado con independencia de sus características individuales. A partir de este clima de respeto a la diversidad, como punto de partida, el profesorado puede trabajar para conseguir el resto de aprendizajes y competencias de la asignatura.

También sería interesante que el profesorado educara al alumnado en una perspectiva crítica de la educación física, el deporte y la salud. En este sentido, se podrían abordar actividades que ayudaran a los jóvenes a identificar los discursos sociales dominantes, las visiones restrictivas de la realidad que generan y las situaciones de injusticia social a las que pueden dar lugar, para llegar a conclusiones finales y posibles soluciones (por ejemplo; diálogos y debates a partir de películas, anuncios o noticias disponibles en la web; trabajos teóricos sobre temas sociocríticos que inciten a la reflexión; actividades prácticas de expresión corporal que simbolicen alguno de estos temas; etc.). Diversas propuestas se han presentado en la literatura al respecto (Aguareles, Domingo, López, Marrón, y Julián, 2015; Devís-Devís y Peiró-Velert, 2004; Gómez y Prat, 2009). No obstante, lo más importante es que el profesorado sirva de ejemplo, evitando y enfrentándose a estos discursos durante el día a día de sus clases prácticas, utilizando un lenguaje apropiado, velando por la inclusión, interviniendo ante casos de marginación y favoreciendo con su labor docente la construcción de identidades positivas, especialmente en aquellos alumnos más vulnerables.

Respecto al uso de pruebas objetivas de valoración de la condición física y la habilidad motriz, los datos de este estudio simplemente alertan de algunos riesgos que pueden comportar. No se trata de afirmar categóricamente que estas pruebas son inadecuadas en el contexto de la educación física, sino de incentivar la reflexión y el debate para que puedan convertirse en recursos educativos interesantes. Por ejemplo, el uso de estas pruebas podría ser apropiado si se plantean en un contexto inclusivo y tolerante, si no ocupan excesivo tiempo de la programación docente, o si se muestran y practican para que el alumnado sepa cómo evaluar su condición física y su progresión en programas de ejercicio. Es decir, si son parte de la evaluación y no necesariamente de la calificación, o si tienen bajo peso en un sistema de 
Beltrán-Carrillo, V. J., y Devís-Devís, J. (2019). El pensamiento del alumnado inactivo sobre sus experiencias negativas en educación física: los discursos del rendimiento, salutismo y masculinidad hegemónica. RICYDE. Revista internacional de ciencias del deporte. 55(15), 20-34. https://doi.org/10.5232/ricyde2019.05502

calificación que ofrece muchas más alternativas para que el alumnado con baja condición física y competencia motriz también puedan sacar buena nota en la asignatura. Se trata de que existan retos motivantes, ajustados a las características e intereses de todo el alumnado, que puedan ser superados con dedicación y esfuerzo, incluso entrenando y realizando ejercicio fuera de clase si fuera necesario. Además, el alumnado vulnerable no debería ser objeto de atención negativa o simplemente el centro de las miradas incómodas del resto de la clase durante la realización de este tipo de pruebas. Con ello no sólo se hace un flaco favor a estos estudiantes, sino al conjunto de la asignatura y la profesión.

Por último, ante los vínculos entre educación física y acoso escolar identificados en la literatura (Durán, 2016; Jachyra, 2016; O’Connor y Graber, 2014), sería interesante que el profesorado de educación física se involucrara activamente en los planes de su centro educativo para la prevención del acoso escolar y la intervención ante este tipo de situaciones. También sería aconsejable la oferta de cursos de formación de profesorado que ofrecieran estrategias para evitar el impacto negativo de ciertos discursos sociales en las clases. Se trata de intentar mejorar los recursos del profesorado para intervenir ante las situaciones de injusticia y marginación que, por desgracia, parecen ser demasiado frecuentes en la escuela.

\section{Referencias}

Adelman, M., \& Ruggi, L. (2008). The beautiful and the abject. Gender, identity and constructions of the body in contemporary Brazilian culture. Current Sociology, 56(4), 555-586. https://doi.org/10.1177/0011392108090942

Aguareles, I.; Domingo, J.; López, S.; Marrón, D., y Julián, J.A. (2015). Desarrollo y evaluación de identidades activas y cívicas. Un modelo pedagógico relacionado con la salud. Tándem. Didáctica de la Educación Física, 50, 32-38.

Albarracín-Pérez, A.; Moreno-Murcia, J.A., y Beltrán-Carrillo, V.J. (2014). La situación actual de la educación física según su profesorado: un estudio cualitativo con profesores de la Región de Murcia. Cultura, Ciencia y Deporte, 9(27), 225-234. https://doi.org/10.12800/ccd.v9i27.469

Atienza, R.; Valencia-Peris, A.; Martos-García, D.; López-Pastor, V. M., y Devís-Devís, J. (2016) La percepción del alumnado universitario de educación física sobre la evaluación formativa: ventajas, dificultades y satisfacción. Movimento, 22(4), 1033-1048. https://doi.org/10.22456/1982-8918.59732

Avery, M., \& Lumpkin, A. (1987). Student's perception of Physical Education objectives. Journal of Teaching in Physical Education, 7, 5-11. https://doi.org/10.1123/jtpe.7.1.5

Ball, S.J. (2003). The teacher's soul and the terrors of performativity. Journal of Education Policy, 18, 215-228. https://doi.org/10.1080/0268093022000043065

Bazeley, P., \& Jackson, K. (2013). Qualitative data analysis with NVivo. London: Sage.

Beltrán-Carrillo, V.J.; Devís-Devís, J., y Peiró-Velert, C. (2012b). Actividad física y sedentarismo en adolescentes de la Comunidad Valenciana. Revista Internacional de Medicina y Ciencias de la Actividad Física y el Deporte, 12(45), 122-137.

Beltrán-Carrillo, V.J.; Devís-Devís, J.; Peiró-Velert, C., \& Brown, D.H.K. (2012a). When physical activity participation promotes inactivity: Negative experiences of Spanish adolescents in physical education and sport. Youth and Society, 44(1), 3-27. https://doi.org/10.1177/0044118X10388262 
Beltrán-Carrillo, V. J., y Devís-Devís, J. (2019). El pensamiento del alumnado inactivo sobre sus experiencias negativas en educación física: los discursos del rendimiento, salutismo y masculinidad hegemónica. RICYDE. Revista internacional de ciencias del deporte. 55(15), 20-34. https://doi.org/10.5232/ricyde2019.05502

Beltrán-Carrillo, V.J.; Devís-Devís, J., \& Peiró-Velert, C. (2018). The influence of body discourses on adolescents' (non)participation in physical activity. Sport, Education and Society, 23(3), 257-269.

https://doi.org/10.1080/13573322.2016.1178109

Bramham, P. (2003). Boys, masculinities and PE. Sport, Education and Society, 8(1), 5771.

https://doi.org/10.1080/1357332032000050060

Braun, V., \& Clarke, V. (2006). Using thematic analysis in psychology. Qualitative Research in Psychology, 3, 77-101. https://doi.org/10.1191/1478088706qp063oa

Brooks, F., \& Magnusson, J. (2006). Taking part counts: Adolescents' experiences of the transition from inactivity to active participation in school-based physical education. Health Education Research, 21, 872-883. https://doi.org/10.1093/her/cyl006

Campos, P.; Saguy, A.; Emsberger, P.; Oliver, E., \& Gaesser, G. (2006). The epidemiology of overweight and obesity: public health crisis or moral panic? International Journal of Epidemiology, 35, 55-60. https://doi.org/10.1093/ije/dyi254

Carlson, T. B. (1995). We hate gym: Student alienation from physical education. Journal of Teaching in Physical Education, 14(4), 467-477. https://doi.org/10.1123/jtpe.14.4.467

Cockburn, C. (2000). Las opiniones de chicas de 13 y 14 a-os sobre la educación física en las escuelas públicas británicas. Estudio realizado en los condados de Hampshire y Cambridgeshire. Apunts: Educación Física y Deportes, 62, 91-101.

Connell, R.W. (2005). Masculinities. Cambridge, MA: Polity.

Crawford, R. (1980). Healthism and the medicalization of everyday life. International Journal of Health Sciences, 10, 365-388. https://doi.org/10.2190/3H2H-3XJN-3KAY-G9NY

Denison, J., \& Scott-Thomas, D. (2011). Michael Foucault: Power and discourse. The 'loaded' language of coaching. En R.L. Jones, P. Potrac, C. Cushion, y LT. Ronglan (Eds.), The sociology of sports coaching (pp.27-39). Abingdon: Routledge.

Devís-Devís, J. (2014). Salutismo. En F.J. González y P.E. Fensterseifer (orgs.) Dicionário crítico de educaçao física (3a ediçao) (pp.590-593). Ijuí: Unijuí.

Devís-Devís, J.; Beltrán-Carrillo, V.J., \& Peiró-Velert, C. (2015). Exploring socio-ecological factors influencing active and inactive Spanish students in years 12 and 13. Sport, Education and Society, 20(3), 361-380. https://doi.org/10.1080/13573322.2012.754753

Devís-Devís, J., y Peiró-Velert, C. (2004). Los materiales curriculares en educación física. En A. Fraile (Ed.) Didáctica de la educación física. Una perspectiva crítica y transversal (pp.63-94). Madrid: Biblioteca Nueva.

Devís-Devís, J.; Pereira-García, S.; López-Cañada, E.; Fuentes-Miguel, J., \& PérezSamaniego, V. (2018). Looking back into trans persons' experiences in heteronormative secondary physical education contexts. Physical Education and Sport Pedagogy, 23(1), 103-116. https://doi.org/10.1080/17408989.2017.1341477

Durán, J. (2016). Acoso y violencia escolar: El papel del profesor de Educación Física y Deportes. Revista Pedagógica Adal, 19(33), 26-31. 
Beltrán-Carrillo, V. J., y Devís-Devís, J. (2019). El pensamiento del alumnado inactivo sobre sus experiencias negativas en educación física: los discursos del rendimiento, salutismo y masculinidad hegemónica. RICYDE. Revista internacional de ciencias del deporte. 55(15), 20-34. https://doi.org/10.5232/ricyde2019.05502

Ennis, C. D. (1996). Students' experiences in sport-based physical education: [more than] Apologies are necessary. Quest, 48, 453-456.

https://doi.org/10.1080/00336297.1996.10484211

Fisette, J. L. (2013). 'Are you listening?': adolescent girls voice how they negotiate selfidentified barriers to their success and survival in physical education. Physical Education and Sport Pedagogy, 18, 184-203.

https://doi.org/10.1080/17408989.2011.649724

Flintoff, A., \& Scraton, S. (2001). Stepping into active leisure? Young women's perceptions of active lifestyles and their experiences of school physical education. Sport, Education and Society, 6(1), 5-21.

https://doi.org/10.1080/713696043

Garrett, R. (2004a). Gendered bodies and physical identities. En J. Evans, B. Davies, \& J. Wright (Eds.), Body knowledge and control. Studies in the sociology of physical education and health (pp.140-156). London: Routledge.

Garrett, R. (2004b). Negotiating a physical identity: Girls, bodies and physical education. Sport, Education and Society, 9(2), 223-237.

https://doi.org/10.1080/1357332042000233958

Gómez, I. y Prat, M. (2009). Hacia una educación física crítica y transformadora (monográfico). Cultura y Educación, 21(1), 9-17. https://doi.org/10.1174/113564009787531190

Graham, G. (2008). Children's and adults' perceptions of elementary school physical education. The Elementary School Journal, 108, 241-249. https://doi.org/10.1086/529106

Hare, M.K., \& Graber, K.C. (2001). Student misconceptions during two invasion game units in physical education: a qualitative investigation of student thought processing. Journal of Teaching in Physical Education, 20(1), 55-77. https://doi.org/10.1123/jtpe.20.1.55

Hopple, C., \& Graham, G. (1995). What children think, feel, and know about physical fitness testing. Journal of Teaching in Physical Education, 14(4), 408-417. https://doi.org/10.1123/jtpe.14.4.408

Jachyra, P. (2016). Boys, bodies, and bullying in health and physical education class: Implications for participation and well-being. Asia-Pacific Journal of Health, Sport and Physical Education, 7(2), 121-138.

https://doi.org/10.1080/18377122.2016.1196112

Johnson, S.; Gray, S., \& Horrell, A. (2013). 'I want to look like that': Healthism, the ideal body and physical education in a Scottish secondary school. Discourse, 34(3), 457-473. https://doi.org/10.1080/01596306.2012.717196

Kenway, J., \& Fitzclarence, L. (1997). Masculinity, violence and schooling: Challenging "poisonous pedagogies." Gender and Education, 9, 117-133.

https://doi.org/10.1080/09540259721493

Kirk, D., \& Colquhoun, D. (1989). Healthism and physical education. British Journal of Sociology of Education, $10(4)$, 417-434. https://doi.org/10.1080/0142569890100403

Lake, J. (2001). Young people's conceptions of sport, physical education and exercise: Implications for physical education and the promotion of health-related exercise. European Physical Education Review, 7, 80-91. https://doi.org/10.1177/1356336X010071003 
Beltrán-Carrillo, V. J., y Devís-Devís, J. (2019). El pensamiento del alumnado inactivo sobre sus experiencias negativas en educación física: los discursos del rendimiento, salutismo y masculinidad hegemónica. RICYDE. Revista internacional de ciencias del deporte. 55(15), 20-34. https://doi.org/10.5232/ricyde2019.05502

Lee, A. M. (1997). Contributions of research on student thinking in physical education. Journal of Teaching in Physical Education, 16, 262-277. https://doi.org/10.1123/jtpe.16.3.262

Lee, A.M.; Carter, J.A., \& Xiang, P. (1995). Children's conceptions of ability in physical education. Journal of Teaching in Physical Education, 14(4), 384-393. https://doi.org/10.1123/jtpe.14.4.384

Li, W., \& Rukavina, P. (2012). The nature, occurring contexts, and psychological implications of weight-related teasing in urban physical education programs. Research Quarterly for Exercise and Sport, 83(2), 308-317. https://doi.org/10.5641/027013612800745130

López-Pastor; V.M., Kirk, D.; Lorente-Catalán, E.; MacPhail, A., \& Macdonald, D. (2013). Alternative assessment in physical education: a review of international literature. Sport, Education and Society, 18(1), 57-76. https://doi.org/10.1080/13573322.2012.713860

López-Pastor, V.M.; Monjas, R.; Gómez, J.; López, E.; Martín, J.; González, J., ... Marugán, L. (2006). La evaluación en educación física. Revisión de modelos tradicionales y planteamiento de una alternativa. La evaluación formativa y compartida. Retos. Nuevas tendencias en Educación Física, Deporte y Recreación, 10, 31-41.

Mansfield, L., \& Rich, E. (2013). Public health pedagogy, border crossings and physical activity at every size. Critical Public Health, 23(3), 356-370.

https://doi.org/10.1080/09581596.2013.783685

Marsi, L. (2007). El pensamiento "economicista", base ideológica del modelo neoliberal. Historia Actual Online, 14, 175-190.

Monforte, J., y Pérez-Samaniego, V. (2017). El miedo en educación física: una historia reconocible. Movimento, 23(1), 85-100.

https://doi.org/10.22456/1982-8918.71272

Moreno-Murcia, J. A.; Rodríguez, P. L. y Gutiérrez, M. (2003). Intereses y actitudes hacia la Educación Física. Revista Española de Educación Física, 9(2), 14-28.

O'Connor, J. A., \& Graber, K.C. (2014). Sixth-grade physical education: An acculturation of bullying and fear. Research Quarterly for Exercise and Sport, 85(3), 398-408. https://doi.org/10.1080/02701367.2014.930403

Olssen, M., \& Peters, M.A. (2005). Neoliberalism, higher education and the knowledge economy: From the free market to knowledge capitalism. Journal of Education Policy, 20, 313-345.

https://doi.org/10.1080/02680930500108718

O'Sullivan, M., \& MacPhail, A. (eds.) (2010). Young people's voices in physical education and sport. London: Routledge.

Peiró-Velert, C.; Valenciano-Valcárcel, J.; Beltrán-Carrillo, V.J., y Devís-Devís, J. (2014). Variabilidad de la actividad física en adolescentes españoles de 17-18 a-os en función del tipo de jornada y época del año. Revista de Psicología del Deporte, 23(2), 347-354.

Peterson, J.L.; Puhl, R.M., \& Luedicke, J. (2012). An experimental assessment of physical educators' expectations and attitudes: The importance of student weight and gender. Journal of School Health, 82(9), 432-440.

https://doi.org/10.1111/j.1746-1561.2012.00719.x 
Beltrán-Carrillo, V. J., y Devís-Devís, J. (2019). El pensamiento del alumnado inactivo sobre sus experiencias negativas en educación física: los discursos del rendimiento, salutismo y masculinidad hegemónica. RICYDE. Revista internacional de ciencias del deporte. 55(15), 20-34. https://doi.org/10.5232/ricyde2019.05502

Piedra, J.; Rodríguez-Sánchez, A.R.; Ries, F., y Ramírez-Macías, G. (2017). Homofobia, heterosexismo y Educación física: Percepciones del alumnado. Profesorado: Revista de curriculum y formación del profesorado, 17(1), 325-338.

Portman, P. (1995). Who is having fun in physical education classes? Experiences of sixthgrade students in elementary and middle schools. Journal of Teaching in Physical Education, 14(4), 445-453.

https://doi.org/10.1123/jtpe.14.4.445

Real Decreto 1105/2014, de 26 de diciembre, por el que se establece el currículo básico de la Educación Secundaria Obligatoria y del Bachillerato. Boletín Oficial del Estado. Madrid, 3 enero 2015, núm. 3, pp.169-546.

Rich, E.; De Pian, L., \& Francombe-Webb, J. (2015). Physical cultures of stigmatisation: Health policy and social class. Sociological Research Online, 20(2). https://doi.org/10.5153/sro.3613

Scraton, S., \& Flintoff, A. (2002). Gender and sport: A reader. London: Routledge.

Shilling, C. (2012). The body and social theory. London: Sage. https://doi.org/10.4135/9781473914810

Sicilia, A. (2003). La investigación sobre el pensamiento del alumnado. Una revisión desde la educación física. Revista de Educación, 331, 577-613.

Smith, B., \& McGannon, K.R. (2017). Developing rigor in qualitative research: Problems and opportunities within sport and exercise psychology. International Review of Sport and Exercise Psychology. Advance online publication. https://doi.org/10.1080/1750984X.2017.1317357

Solmon, M. (2006). Learner cognition. En D. Kirk, M. O'Sullivan, y D. Macdonald (Eds.), The handbook of physical education (pp.226-241). London: Sage. https://doi.org/10.4135/9781848608009.n13

Sparkes, A. C., \& Smith, B. (2014). Qualitative research methods in sport, exercise and health. From process to product. London: Routledge.

Spencer-Cavaliere, N., \& Rintoul, M.A. (2012). Alienation in physical education from the perspectives of children. Journal of Teaching in Physical Education, 31, 344-361. https://doi.org/10.1123/jtpe.31.4.344

Úbeda-Colomer, J.; Monforte, J., y Devís-Devís, J. (2017). Percepción del alumnado sobre una Unidad Didáctica de enseñanza comprensiva de los juegos deportivos de invasión en Educación Física. Retos: Nuevas Tendencias en Educación Física, Deportes y Recreación, 31, 275-281.

Van Daalen, C. (2005). Girls' experiences in physical education: Competition, evaluation, and degradation. The Journal of School Nursing, 21(2), 115-121. https://doi.org/10.1177/10598405050210020901

Walling, M.D., \& Martinek, T.J. (1995). Learned helplessness. Journal of Teaching in Physical Education, 14, 454-466.

https://doi.org/10.1123/jtpe.14.4.454

Wittrock, M.C. (1989). Procesos de pensamiento de los alumnos. En M.C. Wittrock (Ed.), La investigación de la enseñanza, III. Profesores y alumnos (pp.544-626). Barcelona: Paidós/MEC.

\section{Agradecimientos}

Los autores agradecen a la Generalitat Valenciana la ayuda prestada para la realización del proyecto de investigación del cual deriva este artículo (GV04B/176). También agradecen a los escolares adolescentes de Bachillerato su participación en esta investigación. 